\title{
Intermittent, moderate-intensity aerobic exercise for only eight weeks reduces arterial stiffness: evaluation by measurement of stiffness parameter and pressure-strain elastic modulus by use of ultrasonic echo tracking
}

\author{
Midori Tanaka $\cdot$ Motoaki Sugawara $\cdot$ \\ Yasuo Ogasawara · Tadafumi Izumi • \\ Kiyomi Niki · Fumihiko Kajiya
}

Received: 30 July 2012/ Accepted: 24 September 2012/Published online: 15 November 2012

(C) The Japan Society of Ultrasonics in Medicine 2012

\begin{abstract}
Background and purpose Aerobic exercise has been reported to be associated with reduced arterial stiffness. However, the intensity, duration, and frequency of aerobic exercise required to improve arterial stiffness have not been established. In addition, most reports base their conclusions on changes in pulse wave velocity, which is an indirect index of arterial stiffness. We studied the effects of short-term, intermittent, moderate-intensity exercise training on arterial stiffness based on measurements of the stiffness parameter $(\beta)$ and pressure-strain elastic modulus $\left(E_{\mathrm{p}}\right)$, which are direct indices of regional arterial stiffness. Methods A total of 25 young healthy volunteers (18 men) were recruited. By use of ultrasonic diagnostic equipment we measured $\beta$ and $E_{\mathrm{p}}$ of the carotid artery before and after 8 weeks of exercise training.

Results After exercise training, systolic pressure $\left(P_{\mathrm{s}}\right)$, diastolic pressure $\left(P_{\mathrm{d}}\right)$, pulse pressure, systolic arterial diameter $\left(D_{\mathrm{s}}\right)$, and diastolic arterial diameter $\left(D_{\mathrm{d}}\right)$ did not change significantly. However, the pulsatile change in diameter $\left(\left(D_{\mathrm{s}}-D_{\mathrm{d}}\right) / D_{\mathrm{d}}\right)$ increased significantly, and $\beta$ and $E_{\mathrm{p}}$ decreased significantly.
\end{abstract}

M. Tanaka $(\varangle) \cdot$ M. Sugawara · T. Izumi

Faculty of Health Care Sciences, Himeji Dokkyo University,

7-2-1 Kamiohno, Himeji, Hyogo 670-8524, Japan

e-mail: mikko17_2011@mail.goo.ne.jp

Y. Ogasawara · F. Kajiya

Department of Medical Engineering, Kawasaki University

of Medical Welfare, Kurashiki, Japan

K. Niki

Department of Biomedical Engineering, Tokyo City University,

Tokyo, Japan
Conclusions For healthy young subjects, $\beta$ and $E_{\mathrm{p}}$ were reduced by intermittent, moderate-intensity exercise training for only 8 weeks.

Keywords Arterial stiffness $\cdot$ Exercise $\cdot$ Echo tracking

\section{Introduction}

Many studies have been conducted on the association between exercise training and changes in arterial stiffness in different subject groups, for example young healthy, hypertensive, hyperlipidemic, diabetic, and cardiovascular disease groups [1-5]. Most of the studies evaluated changes in arterial stiffness by measuring changes in pulse wave velocity (PWV) [6, 7]. Conventional methods of measuring PWV have been based on two-point measurements, i.e., measurements of the time of travel of the wave over a known distance, e.g., from the carotid artery to the femoral artery and from the upper arm to the ankle. PWV in a region of an artery is mainly related to the stiffness of the arterial wall of that region, and hence, PWV differs with each region in each artery. Therefore, PWV measured over a relatively long distance is integration of regional PWV in each artery involved within that range. Hence, PWV does not represent the stiffness of a particular single artery. In addition, PWV depends on blood pressure. Therefore, for some cases there is no distinction between the decrease in PWV caused by a decrease in arterial stiffness and that caused by a decrease in blood pressure.

The stiffness of a particular artery is obtained by measurement of pulsatile changes in diameter and pulse pressure. There are a few reports of measurements of arterial stiffness indices in carotid and brachial arteries performed by use of ultrasonic machines [8]. A widely used index of 
arterial stiffness is the pressure-strain elastic modulus, which depends on blood pressure. The index regarded as independent of blood pressure is the stiffness parameter, often called the $\beta$ parameter.

The intensity, duration, and frequency of exercise training vary according to the purpose of the study, and improvement in arterial stiffness by exercise training is not a universal finding. One study reported that an acute resistance exercise program increased arterial stiffness in young healthy men [9]; another study reported that 20 weeks of moderate-intensity aerobic exercise training failed to modify arterial stiffness [10].

Considering cardiovascular rehabilitation procedures, we studied the effects of short-term ( 8 weeks), intermittent, moderate-intensity exercise training on arterial stiffness based on measurements of the stiffness parameter and pressure-strain elastic modulus by use of ultrasonic diagnostic equipment.

\section{Materials and methods}

Measurement of arterial stiffness

We measured the pressure-strain elastic modulus $\left(E_{\mathrm{p}}\right)$ and the stiffness parameter $(\beta)$ of the common carotid artery. $E_{\mathrm{p}}$ and $\beta$ are defined as follows:

$E_{\mathrm{p}}=\left(P_{\mathrm{s}}-P_{\mathrm{d}}\right) /\left[\left(D_{\mathrm{s}}-D_{\mathrm{d}}\right) / D_{\mathrm{d}}\right]$

$\beta=\ln \left(P_{\mathrm{s}} / P_{\mathrm{d}}\right) /\left[\left(D_{\mathrm{s}}-D_{\mathrm{d}}\right) / D_{\mathrm{d}}\right]$ where $P_{\mathrm{s}}$ and $P_{\mathrm{d}}$ are, respectively, the systolic and diastolic blood pressures, $D_{\mathrm{s}}$ and $D_{\mathrm{d}}$ are the systolic and diastolic diameters, respectively, of the carotid artery, and ln denotes the natural logarithm.

$\left(D_{\mathrm{s}}-D_{\mathrm{d}}\right) / D_{\mathrm{d}}$ may be called the pulsatile circumferential strain.

\section{Equipment and systems}

For measurement of diameter-change waveforms, we used an echo-tracking system incorporated in ultrasonic diagnostic equipment with a linear array probe (10 or $13 \mathrm{MHz}$ ). The echo-tracking system measured arterial diameter change at a rate of $1 \mathrm{kHz}$ with a precision of $0.01 \mathrm{~mm}$ (Fig. 1). The systolic and diastolic pressures were measured with a cuff-type manometer applied to the right upper arm. The details and reproducibility of this system have been described elsewhere [11].

\section{Subjects}

We studied 25 healthy volunteers (18 men and 7 women, mean age $20.8 \pm 1.0$ years, age range $20-23$ years, body mass index $(B M I)=22.7 \pm 2.9 \mathrm{~kg} \mathrm{~m}^{-2}$ ). Written informed consent was obtained from all the subjects before the study. Approval from the Ethics Committee of Himeji Dokkyo University was obtained before initiation of the study. Subject baseline characteristics are shown in Table 1.
Fig. 1 Measurement of diameter-change waveform. Left long-axis view of the common carotid artery and ultrasound beam steering. By setting the tracking positions displayed as small bars on the echo-tracking beam (line $A$ ) to arterial walls, echo tracking automatically starts. Right the diameterchange waveform, which is calculated by subtracting the distance to the near wall from that to the far wall, is displayed on the M-mode view

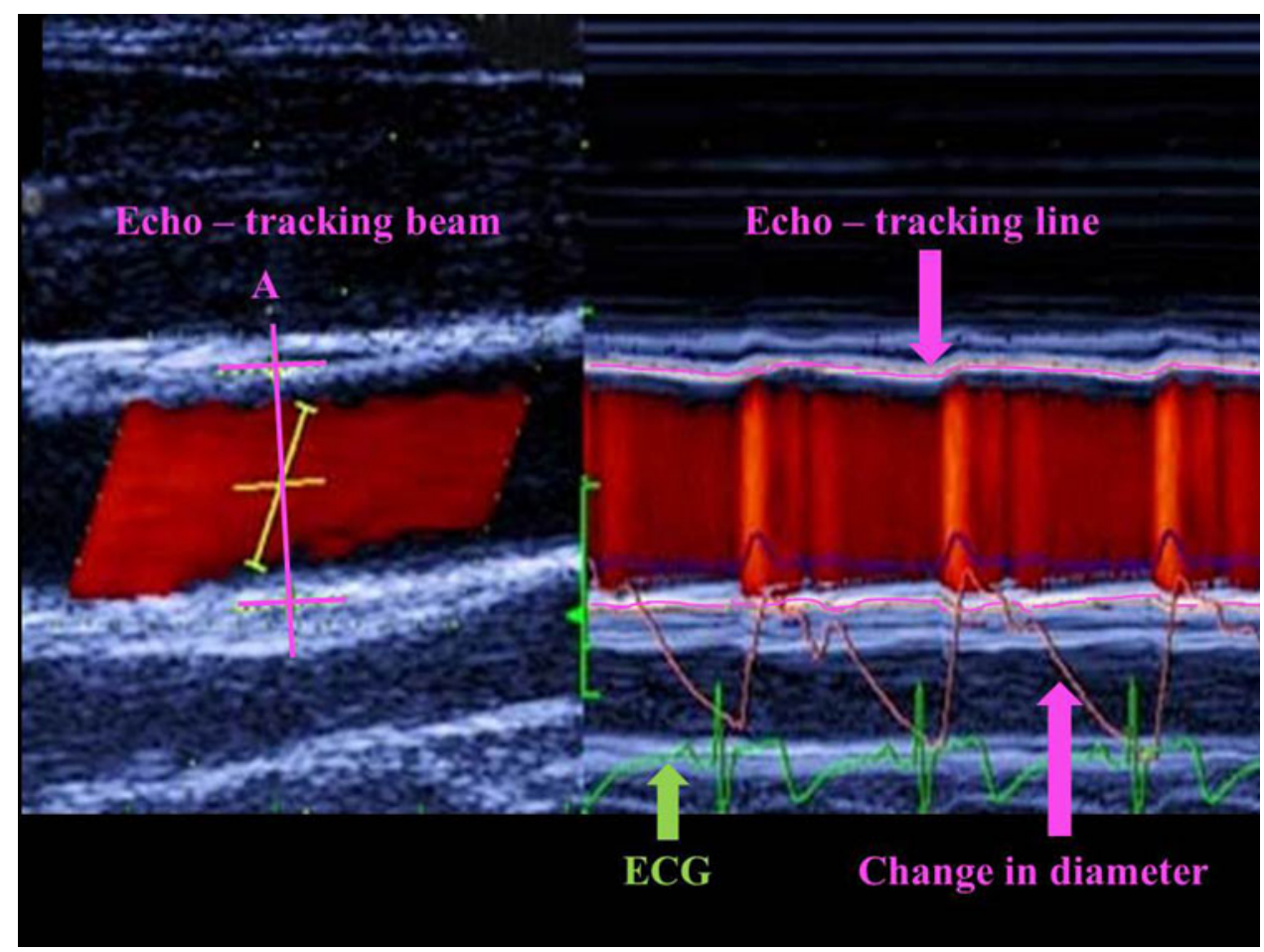


Table 1 Baseline

Characteristics

$B M I$ body mass index, $P_{\mathrm{s}}$ systolic pressure, $P_{\mathrm{d}}$ diastolic pressure, $\beta$ stiffness parameter, $E_{\mathrm{p}}$ pressure-strain elastic modulus

\section{Exercise test procedure}

All measurements were performed by the same observer throughout the study. Subjects were asked to refrain from caffeine, alcohol, and exercise during the 24-h period before each test. The first test consisted of taking of health histories, degree of physical activity, measurements of $E_{\mathrm{p}}$ and $\beta$ at rest, and measurements of peak aerobic capacity (peak $V \mathrm{O}_{2}$ ).

For measurement of $E_{\mathrm{p}}$ and $\beta$, the subjects lay down in the supine position and rested for $10 \mathrm{~min}$ before the first recording was made. The location to be measured was the common carotid artery approximately $2 \mathrm{~cm}$ proximal to the carotid bulb (Fig. 1). We used scanning in the long axis view and obtained a B-mode image of a longitudinal section of the artery. Optimum images were best achieved by positioning and orienting the probe so that clear and parallel delineation of the intima-media complex at both the anterior and posterior walls could be seen. The echotracking beam was steered so that it was orthogonal $\left(90^{\circ}\right)$ to the arterial walls. With the B and M-mode scans displayed simultaneously on a split screen, the echo-tracking system tracked the vessel wall movements to produce displacement waveforms of the anterior and posterior artery walls. This gave the maximum and minimum diameters.

Echo tracking was performed just outside of the intimamedia complex (near the edge of the adventitia side) where stable echo tracking was possible. Data were saved for $20 \mathrm{~s}$. Blood pressure was measured at the time of recording, and the data were entered into the system manually. Five consecutive beats were ensemble-averaged to obtain a representative waveform. The maximum and minimum values of the diameter-change waveform were read, and $E_{\mathrm{p}}$ and $\beta$ were calculated automatically. Measurements were made three times.

After measurement of $E_{\mathrm{p}}$ and $\beta$, peak $V \mathrm{O}_{2}$ was assessed during a graded exercise test on an electrically braked cycle ergometer. This test started with a warm-up consisting of $2 \mathrm{~min}$ of exercise at a $20-\mathrm{W}$ workload, and then the workload was increased by $20 \mathrm{~W} / \mathrm{min}$ until limitation. The limiting criteria for establishment of peak $V \mathrm{O}_{2}$ included a plateau in oxygen consumption with increasing work rate, increase of pulsation to $158 \mathrm{bpm}$, and achievement of maximum fatigue or the impossibility of continuing exercise. In addition, we fixed electrocardiographic limits at an $\mathrm{ST}$ elevation of $0.1 \mathrm{mV}$ and an ST depression of $0.2 \mathrm{mV}$.

Exercise sessions started from the second test. Exercise sessions were conducted two or three times per week over a period of 8 weeks, and subjects had to attend $90 \%$ of all exercise sessions to remain enrolled in the study. The exercise sessions were $30 \mathrm{~min}$ in duration on the bicycle ergometer. Subjects were asked to make an effort to maintain the heart rate during the session at approximately $60 \%$ of that at the peak $V_{2}$ determined during the first maximum exercise test. After 8 weeks of exercise sessions, $E_{\mathrm{p}}$ and $\beta$ at rest were measured in exactly the same way as for the first test.

\section{Statistical analysis}

The results obtained are presented as mean \pm SD. A paired $t$ test was used to evaluate the changes from baseline. A value of $p<0.05$ was considered statistically significant.

\section{Results}

After 8 weeks of exercise training, $P_{\mathrm{s}}, P_{\mathrm{d}}$, and pulse pressure did not change significantly from the baseline values $\left(P_{\mathrm{s}}\right.$ from $112.6 \pm 8.4$ to $111.4 \pm 9.3 \mathrm{mmHg} ; P_{\mathrm{d}}$ from $61.3 \pm 6.3$ to $60.6 \pm 7.7 \mathrm{mmHg}$; pulse pressure from $51.4 \pm 8.9$ to $50.5 \pm 10.0)$. Systolic arterial diameter $\left(D_{\mathrm{s}}\right)$ and diastolic arterial diameter $\left(D_{\mathrm{d}}\right)$ did not change significantly $\left(D_{\mathrm{s}}\right.$ from $7.38 \pm 0.50$ to $7.28 \pm 0.63 \mathrm{~mm} ; D_{\mathrm{d}}$ from $6.78 \pm 0.41$ to $6.63 \pm 0.52 \mathrm{~mm})$. However, $\left(D_{\mathrm{s}}-D_{\mathrm{d}}\right) /$ $D_{\mathrm{d}}$ was significantly increased (from $0.088 \pm 0.020$ to $0.096 \pm 0.021, p<0.01) . \quad \beta$ and $E_{\mathrm{p}}$ were significantly reduced $\left(\beta\right.$ from $7.11 \pm 1.19$ to $6.46 \pm 0.98, p<0.05 ; E_{\mathrm{p}}$ from $79.8 \pm 14.0$ to $71.6 \pm 11.6 \mathrm{kPa}, p<0.05)$. Furthermore, the maximum blood flow velocity in the carotid artery was significantly increased (from $0.83 \pm 0.15$ to $0.89 \pm 0.14 \mathrm{~m} / \mathrm{s}, p<0.05$ ) (Fig. 2).

\section{Discussion}

Integrated arterial stiffness is evaluated by measuring pulse wave velocity (PWV), and several noninvasive methods have been used to assess PWV. However, most of these are associated with problems of accuracy and reproducibility, and none can be considered the "best" method. The coefficient of variation is approximately $8 \%$ at best. This value is obtained by using an MRI system [1, 12], which is impractical in exercise training facilities. Therefore, changes in arterial stiffness because of exercise training 
Fig. 2 Comparison of indices before and 8 weeks after exercise: a systolic pressure $\left(P_{\mathrm{s}}\right), \mathbf{b}$ diastolic pressure $\left(P_{\mathrm{d}}\right)$, c pulse pressure, $\mathbf{d}$ systolic diameter $\left(D_{\mathrm{s}}\right)$, e diastolic diameter $\left(D_{\mathrm{d}}\right)$, $\mathbf{f}$ rate of pulsatile change in diameter $\left(\left(D_{\mathrm{s}}-D_{\mathrm{d}}\right) / D_{\mathrm{d}}\right), \mathbf{g}$ pressurestrain elastic modulus $\left(E_{\mathrm{p}}\right)$, h stiffness parameter $(\beta)$, i maximum velocity (a)

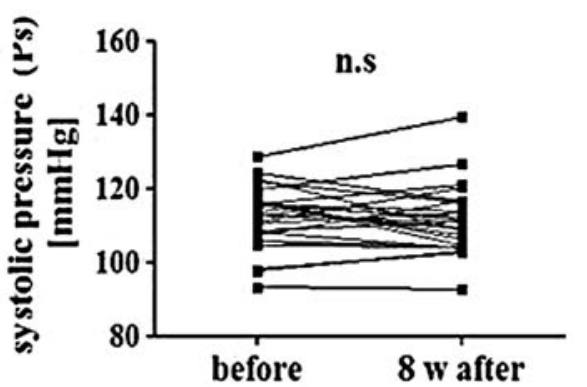

(c)

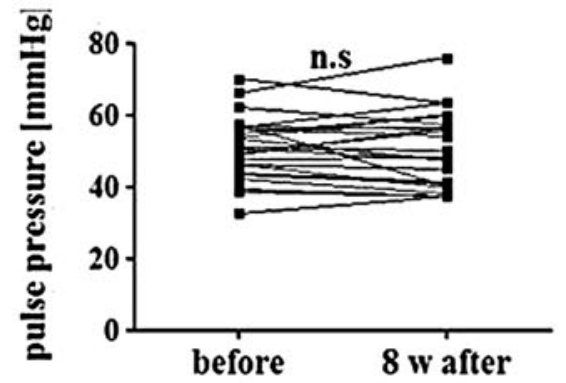

(e)

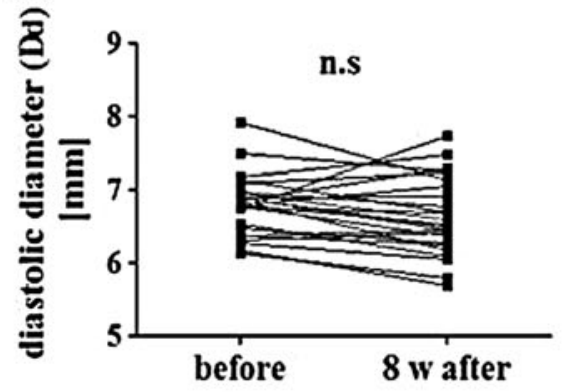

(g)

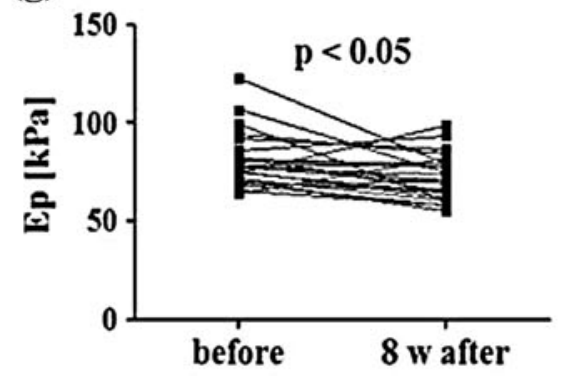

(i)

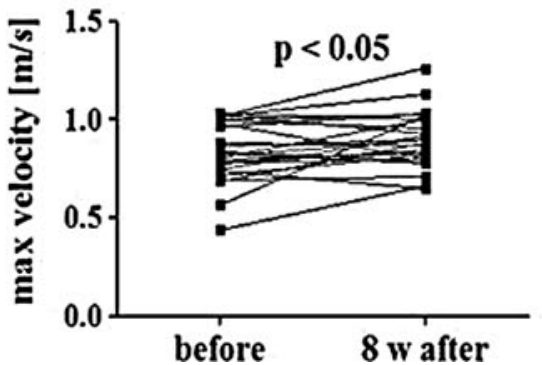

(b)

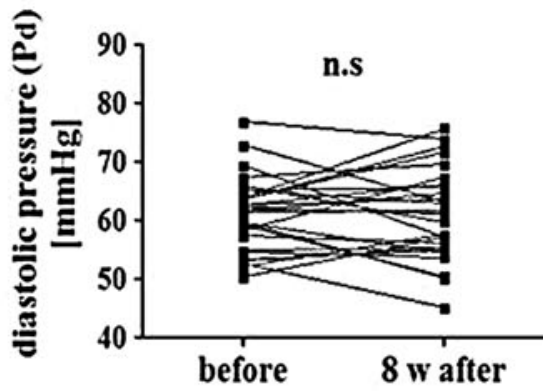

(d)

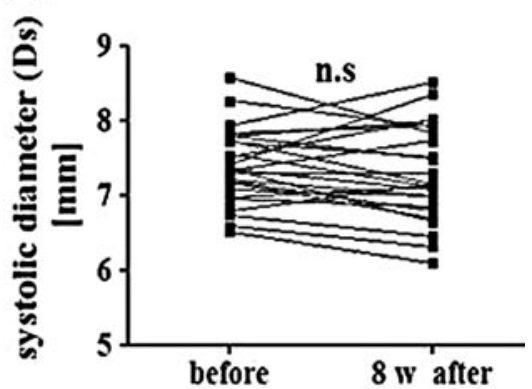

(f)

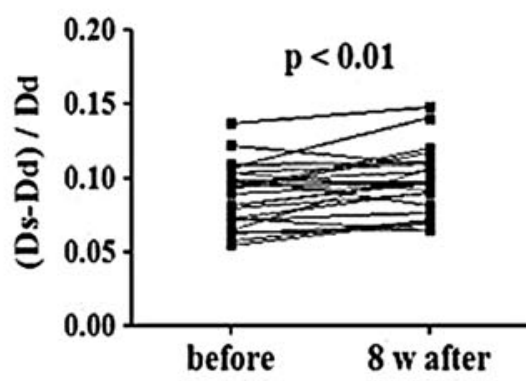

(h)

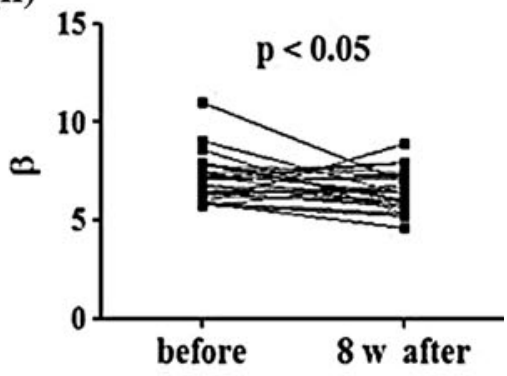


occurring within the space of only 8 weeks is difficult to detect by methods based on PWV measurements.

We measured regional arterial stiffness indices, $E_{\mathrm{p}}$ and $\beta$, by use of ultrasonic echo tracking. Similar to other methods, however, variability of measurements is an inevitable problem with ultrasonic diagnostic equipment. Changes in $E_{\mathrm{p}}$ and $\beta$ after exercise in this study are attributed solely to changes in pulsatile circumferential strain, $\left(D_{\mathrm{s}}-D_{\mathrm{d}}\right) / D_{\mathrm{d}}$. We reported elsewhere [11] that intraobserver intrasession variability of pulsatile circumferential strain was $6.7 \pm 4.0 \%$, and the intraobserver intersession variability of that was $10.8 \%$. Knowing these values of variability, we can compare the reproducibility of different methods. However, it is still difficult to estimate the deviation of each measurement from the true value. Nevertheless, we show a Bland-Altman plot of the measurements of pulsatile circumferential strain before and after exercise training (Fig. 3). Conventional BlandAltman analysis gives the limits of agreement of each pair of measurements. However, what we are especially interested in is the confidence limits for the mean difference (bias). The mean of the percentage differences in strain [100 $\times($ after exercise - before exercise $) /$ average $]$ was $9.18 \%$, and the standard deviation (SD) of the differences was $15.41 \%$. The number of paired measurements $(n)$ was 25 . The standard deviation of the mean difference was $\mathrm{SD} /$ $\sqrt{ } n=15.41 / 5=3.08 \%$. The $95 \%$ confidence limits for the mean difference were $9.18 \pm 1.96 \mathrm{SD} / \sqrt{ } n$, and the $95 \%$ confidence interval was from 3.14 to $15.22 \%$. The line for difference $=0$ (the abscissa) is not contained in the confidence interval, which means that the mean difference between the measurements before and after exercise is significant. A Bland-Altman plot shows graphically but informally how the two measurements differ. More formal statement of the significance level was given by use of the paired Student's $t$ test (Fig. 2f).

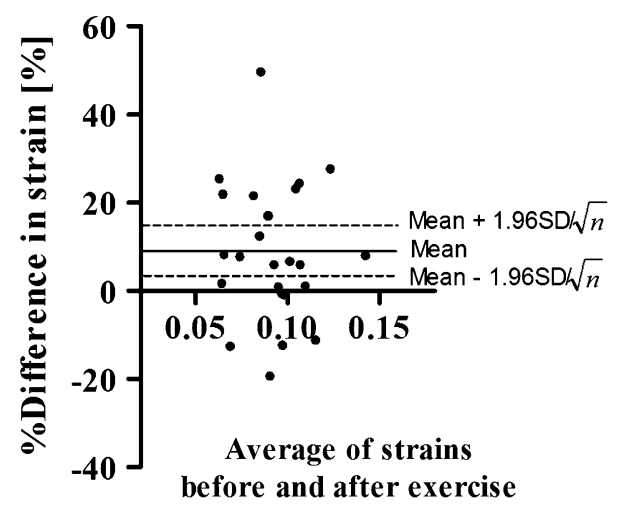

Fig. 3 Intraobserver intersession variability of the pulsatile circumferential strain, $\left(D_{\mathrm{s}}-D_{\mathrm{d}}\right) / D_{\mathrm{d}}$, by Bland-Altman plot. The horizontal solid line indicates the mean difference between the measurements before and after exercise. The two horizontal dotted lines indicate the $95 \%$ confidence limits of the mean difference
Responses of the arterial wall to exercise may differ with each artery, ranging from elastic to muscular arteries. From the perspective of ventriculo-arterial interaction, we are particularly interested in arteries with Windkessel function, the most representative of which is the aorta. However, it is difficult to measure pulsatile changes in aortic diameter by echo tracking. Therefore, we selected the common carotid artery, which also has Windkessel function, as the site for ultrasonic measurements.

$E_{\mathrm{p}}$ is the most commonly used index of arterial stiffness, which is defined on the assumption that the relationship between pressure and diameter change is linear (Eq. 1). However, the relationship between pressure and diameter changes is not linear. Therefore, $E_{\mathrm{p}}$ is affected by changes in pressure, i.e., $E_{\mathrm{p}}$ increases with increasing pressure. On the other hand, $\beta$ is defined on the assumption that the relationship between pressure and diameter changes is semilogarithmic (Eq. 2). This makes $\beta$ relatively independent of pressure [13].

The mechanical basis of the observed change in PWV requires consideration of the relationship between PWV and arterial pressure. Changes in PWV occurring in the presence of arterial pressure changes may simply be a consequence of the nonlinearity of the arterial pressurediameter relationship rather than an intrinsic change in arterial wall properties. The same applies to $E_{\mathrm{p}}$. In this study, the pulse pressure did not change from baseline after exercise training, but the relative pulsatile change in diameter, i.e., pulsatile circumferential strain, $\left(D_{\mathrm{s}}-D_{\mathrm{d}}\right) /$ $D_{\mathrm{d}}$, increased significantly. Therefore, the decreases in $\beta$ and $E_{\mathrm{p}}$ suggest a true change in arterial wall properties.

The use of upper arm pressure, instead of carotid pressure, to obtain $\beta$ and $E_{\mathrm{p}}$ of the carotid artery may be criticized, because arterial pressure is amplified in transit from the ascending aorta to peripheral sites, particularly for young subjects. However, there is a method for obtaining carotid arterial pressure from upper arm pressure. Although the peak pressure is altered as the pressure wave travels, it has been observed that the pressure averaged over a cardiac cycle is constant throughout the extensive arterial tree, and that diastolic pressure (the minimum pressure) does not change substantially. On the basis of these facts, and using the measured arterial diameter-change waveforms as surrogates for pressure waveforms, we can obtain carotid arterial pressure from upper arm pressure. The method is described in detail elsewhere [14]. However, because the method is too timeconsuming to be practical, we did not apply the method in this study. Nevertheless, the measured $P_{\mathrm{s}}, P_{\mathrm{d}}$, and pulse pressure did not change from baseline after the exercise training. Therefore, the method of measuring pressure does not affect the result that $\beta$ and $E_{\mathrm{p}}$ changed after the exercise training. 
The mechanisms underlying the effects of aerobic exercise on arterial stiffness are largely unknown. Nevertheless, it has been suggested that the ability of exercise to change arterial stiffness may reflect vascular structural remodeling associated with increased nitric oxide bioactivity via shear-stress stimulation of endothelial nitric oxide synthase $[1-5,15,16]$. Indeed, the maximum carotid arterial blood velocity after the exercise training increased significantly (Fig. 2i), which caused an increase in shear stress on the luminal surface of the endothelium.

\section{Limitation}

We could not show any direct evidence of the cellular and molecular mechanisms responsible for changes in arterial stiffness caused by exercise training because we had no means of physically accessing human arteries in situ and experimentally manipulating potential signaling pathways.

\section{Conclusions}

In healthy young subjects, $\beta$ and $E_{\mathrm{p}}$, which are indices of arterial stiffness, were improved (reduced) by only 8 weeks of intermittent, moderate-intensity exercise training.

Conflict of interest We declare that we have no conflict of interest in connection with this paper.

\section{References}

1. Arena R, Fei DY, Arrowood JA, Kraft KA. Influence on aerobic fitness on aortic stiffness in apparently healthy Caucasian and African-American subjects. Int J Cardiol. 2007;122:202-6.

2. Madden KM, Lockhart C, Cuff D, Potter TF, Meneilly GS. Shortterm aerobic exercise reduces arterial stiffness in older adults with type 2 diabetes, hypertension, and hypercholesterolemia. Diabetes Care. 2009;32:1531-5.

3. Seals DR, Walker AE, Pierce GL, Lesniewski LA. Habitual exercise and vascular ageing. J Physiol. 2009;587:5541-9.

4. Cameron JD, Dart AM. Exercise training increases total systemic arterial compliance in humans. Am J Physiol. 1994;266:H693-701.

5. Hambrecht R, Fiehn E, Weigl C, Gielen S, Hamann C, Kaiser R, et al. Regular physical exercise corrects endothelial dysfunction and improves exercise capacity in patients with chronic heart failure. Circulation. 1998;98:2709-15.

6. Liang YL, Gatzka CD, Du XJ, Cameron JD, Kingwell BA, Dart AM. Effects of heart rate on arterial compliance in men. Clin Exp Pharmacol Physiol. 1999;26:342-6.

7. Williams MR, Westerman RA, Kingwell BA, Paige J, Blombery PA, Sudhir K, et al. Variations in endothelial function and arterial compliance during the menstrual cycle. J Clin Endocrinol Metab. 2001;86:5389-95.

8. Harada A, Okada T, Niki K, Chang D, Sugawara M. On-line noninvasive one-point measurements of pulse wave velocity. Heart Vessels. 2002;17:61-8.

9. Yoon ES, Jung SJ, Cheun SK, Oh YS, Kim SH, Jae SY. Effects of acute resistance exercise on arterial stiffness in young men. Korean Circ J. 2010;40:16-22. Epub 2010/01/30.

10. Aizawa K, Petrella RJ. Acute and chronic impact of dynamic exercise on arterial stiffness in older hypertensives. Open Cardiovasc Med J. 2008;2:3-8.

11. Niki K, Sugawara M, Chang D, Harada A, Okada T, Sakai R, et al. A new noninvasive measurement system for wave intensity: evaluation of carotid arterial wave intensity and reproducibility. Heart Vessels. 2002;17:12-21. Epub 2002/11/16.

12. Kraft KA, Itskovich VV, Fei DY. Rapid measurement of aortic wave velocity: in vivo evaluation. Magn Reson Med. 2001;46: 95-102. Epub 2001/07/10.

13. Hayashi K, Nagasawa S, Naruo Y, Okamura A, Moritake K, Handa H. Mechanical properties of human cerebral arteries. Biorheology. 1980;17:211-8.

14. Sugawara M, Niki K, Ohte N, Okada T, Harada A. Clinical usefulness of wave intensity analysis. Med Biol Eng Comput. 2009;47:197-206.

15. Tanaka H, Dinenno FA, Monahan KD, Clevenger CM, DeSouza CA, Seals DR. Aging, habitual exercise, and dynamic arterial compliance. Circulation. 2000;102:1270-5.

16. Joyner MJ. Effect of exercise on arterial compliance. Circulation. 2000;102:1214-5. 